\title{
Eficacia del uso de bromuro de ipratropio tópico para el control de la rinorrea en pacientes con rinitis alérgica en comparación con placebo en el Hospital Juárez de México
}

\section{Efficacy of the use of topical ipratropium bromide for rinorrea control in patients with allergic rinitis compared to placebo at Hospital Juárez de México}

\author{
Jonathan A. Velázquez-Peña ${ }^{1 *}$ y Juan F. Gutiérrez-Piedra ${ }^{1,2}$ \\ ${ }^{1}$ Departamento de Cirugía, Otorrinolaringología y Cirugía de Cabeza y Cuello, Universidad Nacional Autónoma de México; ${ }^{2} S e r v i c i o ~ d e$ \\ Otorrinolaringología y Cirugía de Cabeza y Cuello, Hospital Juárez de México. Ciudad de México, México
}

\begin{abstract}
Resumen
Antecedentes: La rinitis alérgica (RA) se encuentra entre las enfermedades más comunes en todo el mundo. Los síntomas clásicos de la RA son prurito nasal, estornudos en salva, rinorrea hialina y congestión nasal. Se ha demostrado la efectividad del uso de bromuro de ipratropio intranasal en el control de la rinorrea en presentación intranasal, sin embargo no contamos con la venta del medicamento en dicha presentación en nuestro país, motivo por el cual decidimos realizar este estudio utilizando la presentación para nebulizar. Métodos: Este ensayo comparó la eficacia y la seguridad del bromuro de ipratropio en solución para nebulizar $250 \mathrm{mcg} / \mathrm{ml}$ tópica intranasal con placebo durante un periodo de tratamiento de cuatro semanas en 60 pacientes con RA y rinorrea como síntoma predominante. Resultados y conclusiones: El bromuro de ipratropio en dosis de 42 mcg por cada fosa nasal tres veces al día, fue bien tolerado por los pacientes durante el mes de estudio y disminuyó los síntomas manifestados por los pacientes previo a su utilización. Con un intervalo de confianza del $99 \%, p<0.001$, siendo muy similar su eficacia como lo reportado en la bibliografía en comparación con el bromuro de ipratropio en su presentación de espray nasal.
\end{abstract}

Palabras clave: Rinitis. Ipratropio. Intranasal. Rinorrea.

\begin{abstract}
Background: Allergic rhinitis $(A R)$ is among the most common diseases worldwide. Classic symptoms of AR are itching, sneezing in salvo, hyaline rhinorrhea and nasal congestion. The effectiveness of the use of intranasal ipratropium bromide in the control of rhinorrhea in intranasal presentation has been demonstrated, however, we do not have the sale of the drug in such presentation in our country, which is why we decided to carry out this study using the presentation to nebulize. Methods: This trial compared the efficacy and safety of ipratropium bromide in solution to mist $250 \mathrm{mcg} / \mathrm{ml}$ topical intranasal with placebo over a 4-week treatment period in 60 patients with AR and rhinorrhea as a predominant symptom. Results and conclusions: Ipratropium bromide at doses of $42 \mathrm{mcg}$ per nostril three times daily, was well tolerated by patients during the month of study and I am alert the symptoms manifested by patients prior to use. With a confidence interval of $99 \%, p<0.001$, being very similar its effectiveness as reported in the literature compared to ipratropium bromide in its presentation of nasal spray.
\end{abstract}

Key words: Rhinitis. Ipratropium. Intranasal. Rhinorrhea.

Correspondencia:

*Jonathan A. Velázquez-Peña

E-mail: axlvelazquez91@gmail.com licencia CC BY-NC-ND (http://creativecommons.org/licenses/by-nc-nd/4.0/).

Fecha de recepción: 23-08-2020

Fecha de aceptación: 18-09-2020

DOI: 10.24875/RHJM.20000098
Disponible en internet: 14-01-2021 Rev Hosp Jua Mex. 2020;87(4):167-175

www.revistahospitaljuarez.com 


\section{Introducción}

La rinitis alérgica (RA) se encuentra entre las enfermedades más comunes en todo el mundo y generalmente persiste durante toda la vida. Se ha estimado que la prevalencia de RA autoinformada es aproximadamente del 2 al $25 \%$ en niños y del 1 a más del $40 \%$ en adultos. La prevalencia de RA confirmada en adultos en Europa osciló entre el 17 y el $28.5 \%$. Estudios recientes muestran que la prevalencia de RA ha aumentado en particular en países con baja prevalencia inicial. Los síntomas clásicos de RA son prurito nasal, estornudos en salva, rinorrea hialina y congestión nasal. Los síntomas oculares también son frecuentes. La RA se asocia con prurito nasal, hiperemia conjuntival y epífora. Otros síntomas incluyen prurito en el paladar, goteo posnasal y tos ${ }^{1,2}$.

La rinorrea asociada con el resfriado común y las alergias se deriva de las glándulas serosas y submucosas de la nariz que secretan moco cuando se activan mediante un estímulo que activa los nervios parasimpáticos que los inervan. El agente anticolinérgico tópico en aerosol nasal de bromuro de ipratropio al $0.06 \%$ inhibe la vía parasimpática nasal. Cuando se aplica localmente, el bromuro de ipratropio inhibe las secreciones de las glándulas serosas y seromucosas que recubren la mucosa nasal ${ }^{2-4}$.

El bromuro de ipratropio es un anticolinérgico que actúa contra el sistema parasimpático a nivel nasal. Al igual que otros medicamentos intranasales, se utilizó por primera vez en el tratamiento de enfermedades bronquiales, como broncodilatador. Estudios posteriores han demostrado que el ipratropio inhibe la hipersecreción nasal inducida por la metacolina, reduce la secreción nasal en pacientes con rinitis perenne y el resfriado común ${ }^{5-7}$.

Los pacientes alérgicos suelen describir la rinorrea como uno de sus síntomas más problemáticos junto con la congestión. Se ha demostrado que el bromuro de ipratropio es eficaz para reducir tanto la gravedad como la duración de la rinorrea en la RA y la rinitis no alérgica con efectos secundarios mínimos. Se ha demostrado que el aerosol nasal es de acción rápida, inhibiendo las secreciones nasales inducidas por metacolina en 15 minutos. No ha habido indicios de rebote nasal con la interrupción del aerosol nasal de bromuro de ipratropio, ni ninguna disminución de la eficacia en estudios a largo plazo hasta un año. Aunque la efectividad del aerosol nasal de ipratropio $0.03 \%$ en el control de la rinorrea está bien establecida, su papel en la práctica clínica aún no se ha definido ${ }^{8-11}$.
El tratamiento adecuado de la RA mejora los síntomas, la calidad de vida y el rendimiento laboral y escolar ${ }^{12-14}$.

Se ha demostrado la efectividad del uso de bromuro de ipratropio intranasal en el control de la rinorrea en presentación intranasal, sin embargo no contamos con la venta del medicamento en dicha presentación en nuestro país, motivo por el cual decidimos realizar este estudio utilizando la presentación para nebulizar; y así evaluar su eficacia, la mejoría de la calidad de vida en pacientes con RA y los efectos adversos de la aplicación tópica del bromuro de ipratropio.

\section{Objetivos}

Comparar la eficacia del bromuro de ipratropio en solución para nebulizar en aplicación tópica nasal vs. placebo en el control de la rinorrea, evaluar la mejoría de la calidad de vida en pacientes con RA e identificar los efectos adversos de la aplicación tópica del bromuro de ipratropio.

\section{Materiales y métodos}

Este ensayo comparó la eficacia y la seguridad del bromuro de ipratropio en solución para nebulizar $250 \mathrm{mcg} / \mathrm{ml}$ tópica intranasal con placebo durante un periodo de tratamiento de cuatro semanas en 60 pacientes con RA y rinorrea como síntoma predominante, quienes se encontraban con tratamiento a base de esteroides nasales. Los pacientes usaron tres gotas de bromuro de ipratropio en solución para nebulizar en cada fosa nasal tres veces al día, correspondiente a $42 \mathrm{mcg}$ de fármaco activo o vehículo salino placebo. Este fue un estudio doble ciego, multicéntrico, aleatorizado, de grupos paralelos con pacientes adultos de edad 18 a 75 años que exhibieron rinorrea clínicamente significativa como un componente principal de la RA. Se excluyeron del estudio pacientes embarazadas, en lactancia, con antecedente de glaucoma, patología prostática o vesical, así como con sensibilidad a la atropina o bromuros.

La rinorrea nasal anterior clínicamente significativa fue definida como sigue: a) presencia de rinorrea por más de una hora diaria al menos cuatro días por semana en el mes anterior; b) presencia de rinorrea de dos a tres semanas por mes durante seis meses del año anterior, y c) una gravedad mínima de las secreciones nasales «leves». Este último parámetro fue calificado por los pacientes en la siguiente escala de síntomas de seis puntos: $0=$ ninguno, $1=$ muy leve, $2=$ leve, $3=$ moderado, $4=$ severo y $5=$ muy grave. Se realizó el test de 
Tabla 1. Cuestionario SNOT 20 (Sino-Nasal Outcome Test de 20 indicadores)

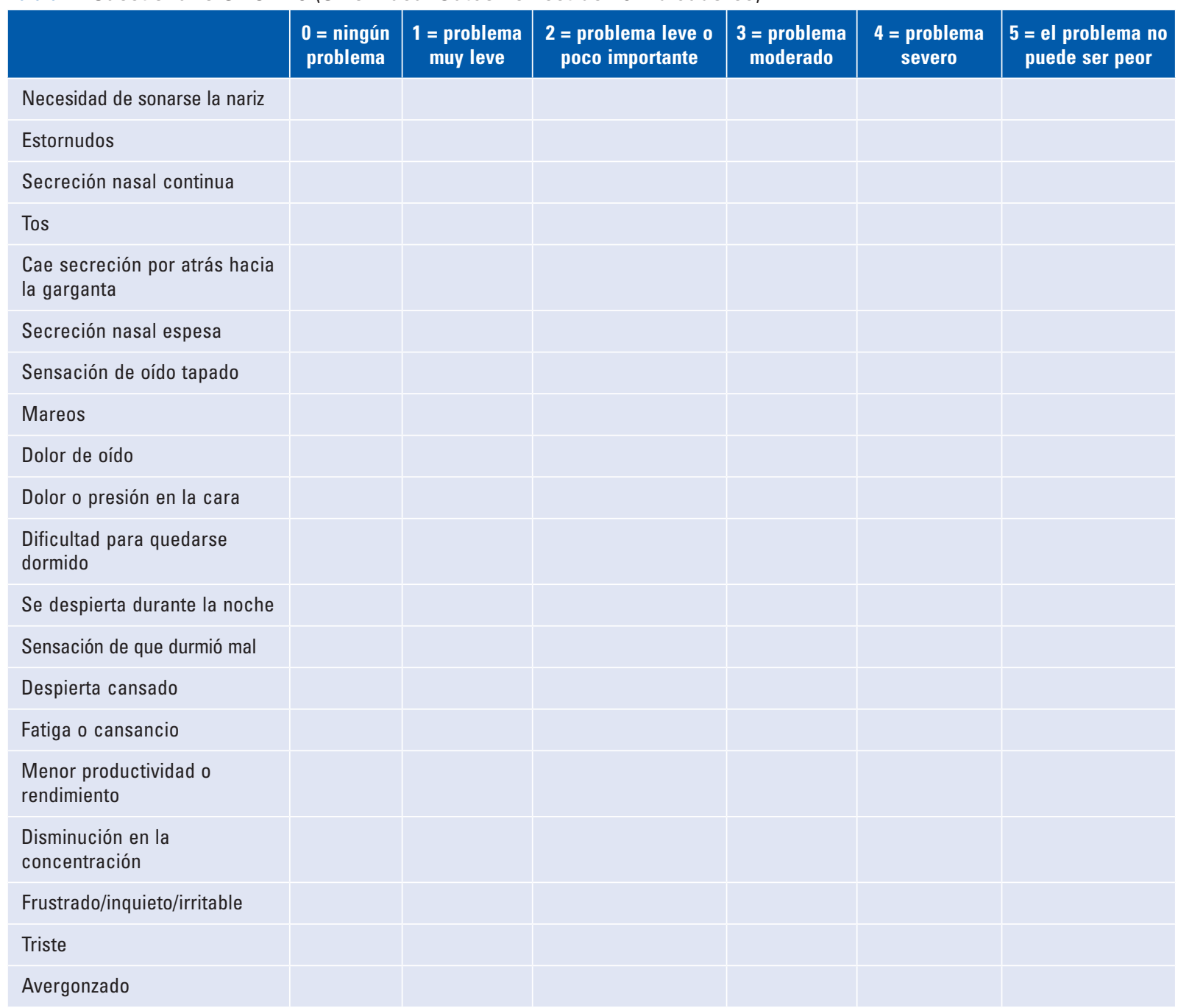

calidad de vida SNOT (Sino-Nasal Outcome Test de 20 indicadores) antes del tratamiento y a las cuatro semanas de este (Tabla 1).

Se realizó un estudio doble ciego, aleatorizado, en el cual se eligieron dos grupos, A y B. En el grupo A, que constaba de 30 pacientes, se utilizó bromuro de ipratropio en solución para nebulizar de manera tópica intranasal y en el grupo B, de 30 pacientes, se utilizó solución fisiológica al $0.9 \%$ en la misma dosis, tres gotas cada ocho horas en cada fosa nasal durante cuatro semanas.

\section{Justificación}

No existe la presentación en nuestro país de bromuro de ipratropio en espray nasal para el tratamiento de la rinorrea, sin embargo ya se cuentan con estudios en los que el bromuro de ipratropio en espray nasal es eficaz en el control de la rinorrea. Al no contar con dicha presentación, recurrimos a la presentación en solución para nebulizar, para comprobar su eficacia en el control de la rinorrea.

\section{Resultados}

Setenta pacientes fueron seleccionados para participar en el estudio y 60 fueron incluidos finalmente, dado que cumplieron con los criterios de selección. Las exclusiones se debieron a que incluyeron medicamentos concomitantes, enfermedad, se perdió el seguimiento o razones personales de los pacientes. 


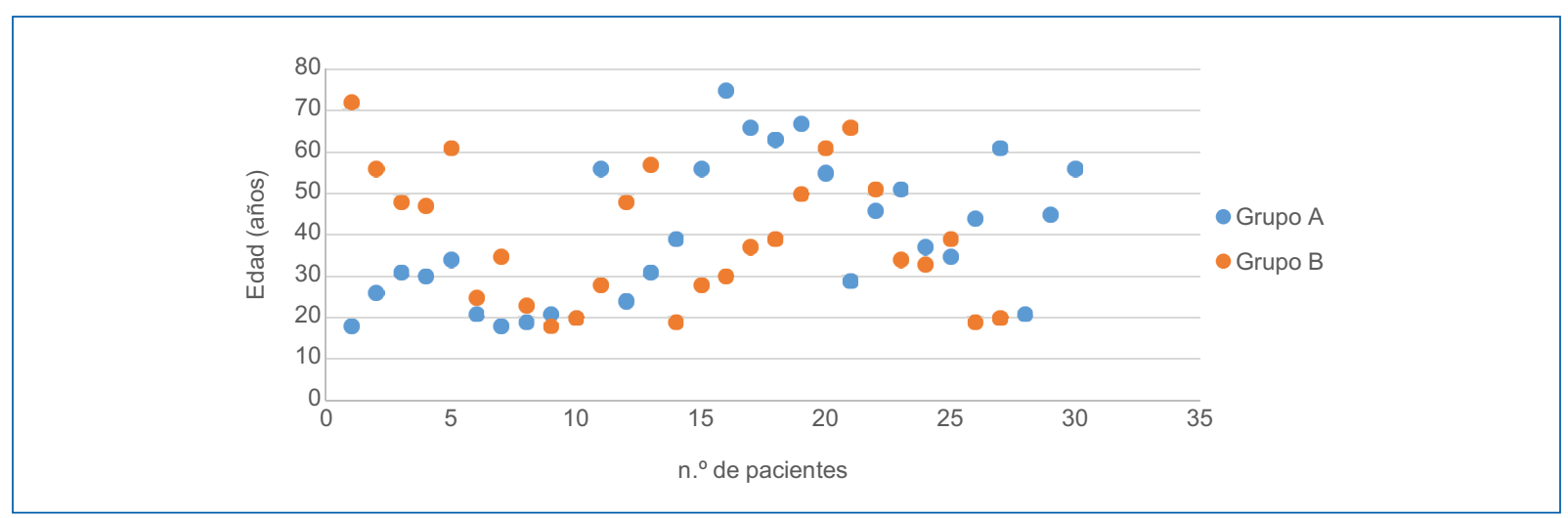

Figura 1. Edad de los pacientes para cada grupo analizado.

De los 60 pacientes seleccionados al azar, 56 completaron el estudio: con un paciente se perdió la comunicación, un paciente no continuó por efectos adversos y dos pacientes no continuaron por no poder dar seguimiento, por lo que se excluyeron del estudio. Se observó sintomatología nasal de moderada a grave en el $96 \%$ de los pacientes con rinorrea.

Los 56 pacientes se dividieron en dos grupos, en el grupo A se analizaron 30 pacientes, con tratamiento de bromuro de ipratropio en solución para nebulizar de manera tópica intranasal y en el grupo $B$ se estudiaron 27 pacientes con solución fisiológica al $0.9 \%$. En ambos grupos se administraron 3 gotas de solución cada 8 horas en cada fosa nasal durante cuatro semanas. Dada la evaluación clínica de rinorrea nasal, esta es: a) presencia de rinorrea por más de una hora diaria al menos cuatro días por semana en el mes anterior; b) presencia de rinorrea de dos a tres semanas por mes durante seis meses del año anterior, y c) una gravedad mínima de las secreciones nasales «leves». La gravedad fue calificada por los pacientes en la siguiente escala de síntomas: 0 = ningún síntoma, 1 = síntomas muy leves, 2 = síntomas leves, 3 = síntomas moderados, $4=$ síntomas severos y $5=$ síntomas muy graves. Además de aplicarse la escala de evaluación de la gravedad de los síntomas por el paciente, también se aplicó el test de calidad de vida SNOT, ambos en los mismos periodos de evaluación: en el pretratamiento, siete días después del tratamiento y cuatro semanas después de este. Del grupo A se tiene un promedio de edad de 38 años \pm 17.0882 y para el grupo $\mathrm{B}$ el promedio de edad corresponde a 39 años \pm 16.0582 ; la edad para cada paciente de ambos grupos se muestra en la figura 1. En la figura 2 se muestra la distribución por género para cada grupo analizado; se puede observar que predomina el sexo femenino con una relación 1.5 mujeres con respecto a los hombres para el caso del grupo A. En el grupo $B$, la relación es de 1.7 mujeres por cada hombre del estudio. En la figura 3 se muestra la relación de la escala de síntomas indicada por los pacientes para cada grupo. Para el grupo A, pacientes que fueron tratados con bromuro de ipratropio, se observa una disminución en la escala de síntomas; para el periodo de pretratamiento se comienza con valores altos (síntomas muy graves) y después de cuatro semanas, la escala disminuye hasta a valores de cero, es decir, sin síntomas. Además, en el grupo A se observa que el bromuro de ipratropio demostró ser de acción rápida, dado que se muestra mejoría durante los primeros siete días del tratamiento. Sin embargo, para el grupo B se puede observar que pasado el tiempo del tratamiento, un mínimo de pacientes refiere mejoría con respecto al inicio del tratamiento o una disminución en la escala de síntomas.

En general, hubo una reducción en la gravedad de la rinorrea para el grupo A en 24 de los 30 pacientes activos en tratamiento, en comparación con el grupo $\mathrm{B}$, donde solo siete de los 26 pacientes mostraron mejoría en los primeros siete días después del tratamiento; ese comportamiento también se manifestó en el cuestionario SNOT, como se puede observar en la figura 4. La escala de calificación de la rinorrea y el cuestionario SNOT para cada paciente se realizó nuevamente a las cuatro semanas, obteniendo una mejoría para el grupo A en 28 de los 30 pacientes de una escala inicial de 3-4 a 0-1, además de una mejoría en la calidad de vida del cuestionario SNOT. En el grupo $B$, solo tres pacientes que presentaban una calificación inicial de 4 indicaron una mejoría, registrando una 
Sexo de los pacientes del grupo A tratados con bromuro de ipatropio

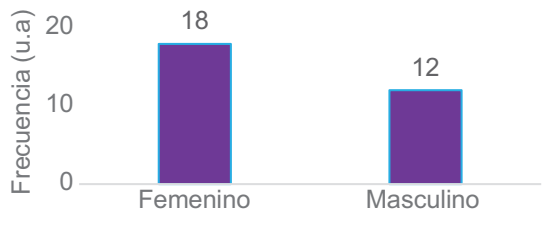

Sexo de los pacientes del grupo $\mathrm{B}$ tratados con solución fisiológica al $0.9 \%$

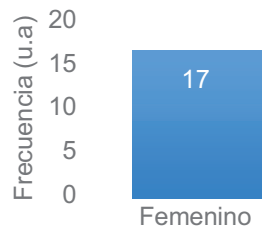

Figura 2. Número de pacientes de sexo femenino o masculino para cada grupo analizado.

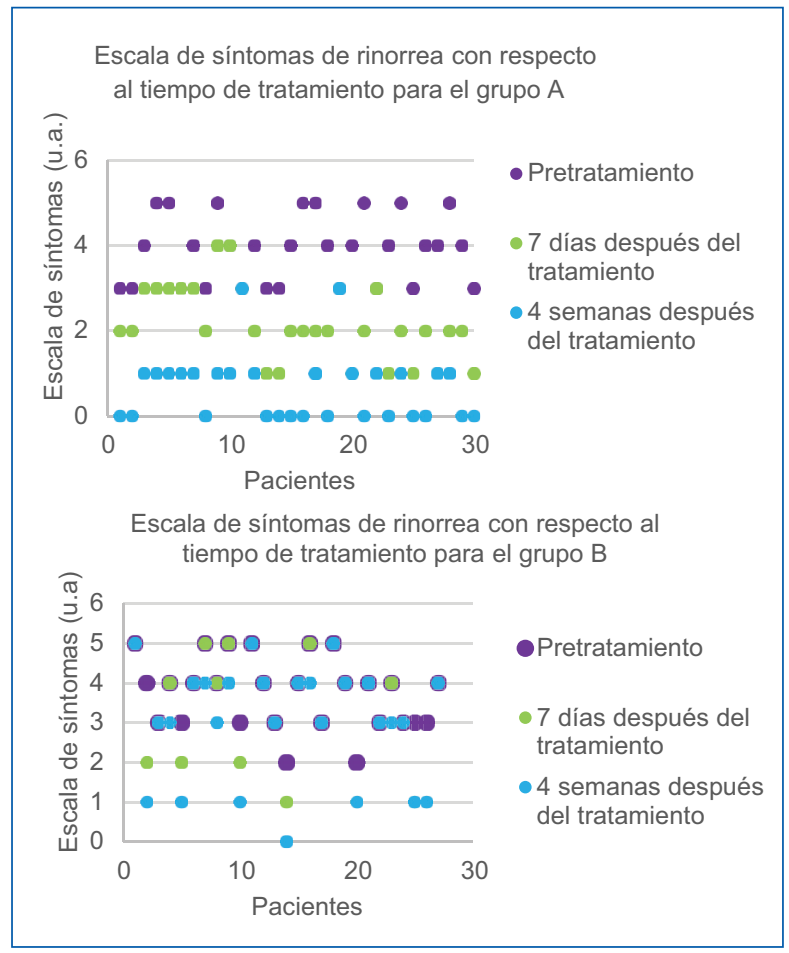

Figura 3. Escala de síntomas para cada grupo analizado durante el pretratamiento, 7 días y 4 semanas después del tratamiento.

escala de 1; seis pacientes que presentaban una calificación de 4, mejoraron a una escala de 2, así mismo al realizarles el cuestionario SNOT solo siete pacientes indicaron mejoría en la calidad de vida. En la figura 5 se compara la mejoría de los pacientes referida con respecto a los periodos de tratamiento para la escala de síntomas indicada por el paciente, así como para la escala en su calidad de vida evaluada en el cuestionario SNOT, comparando tanto el grupo A tratado con bromuro de ipratropio con el grupo $B$ tratado con solución fisiológica. El comportamiento es muy parecido en ambos grupos, con valores de escala similares,

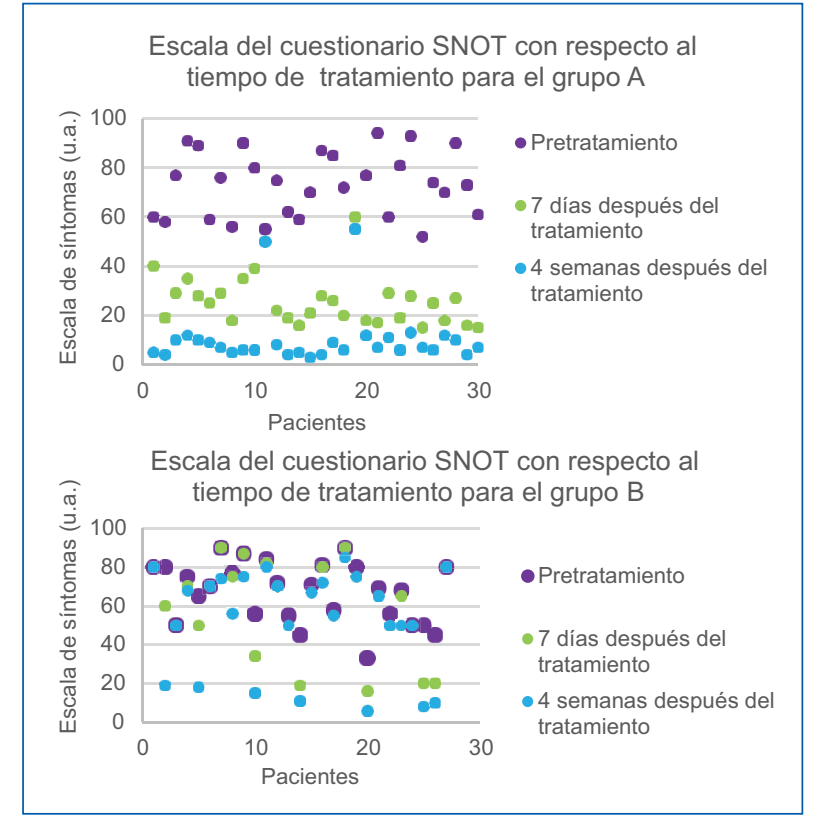

Figura 4. Escala de síntomas para cada grupo analizado durante el pretratamiento, 7 días y 4 semanas después del tratamiento, en el cuestionario SNOT (Sino-Nasal Outcome Test de 20 indicadores).

poca o mínima mejoría manifestada por los pacientes. En el caso de siete días posteriores al comienzo del tratamiento, en el grupo A se muestra una mejoría en la escala de síntomas, con evaluaciones menores a 3 , solo el $6.66 \%$ de los pacientes muestra valores mayores a 4 en la escala de síntomas. Para el grupo B, el $55.55 \%$ de los pacientes muestra valores superiores a 4 en la escala. El mismo comportamiento se observa en los resultados generados por el cuestionario SNOT donde refleja la calidad de vida, para el grupo A solo el $6.66 \%$ indica una escala $\geq 40$ puntos contrario al grupo $B$, donde se muestra que el $81.48 \%$ de los pacientes dan una calificación en la escala $\geq 40$ puntos. 


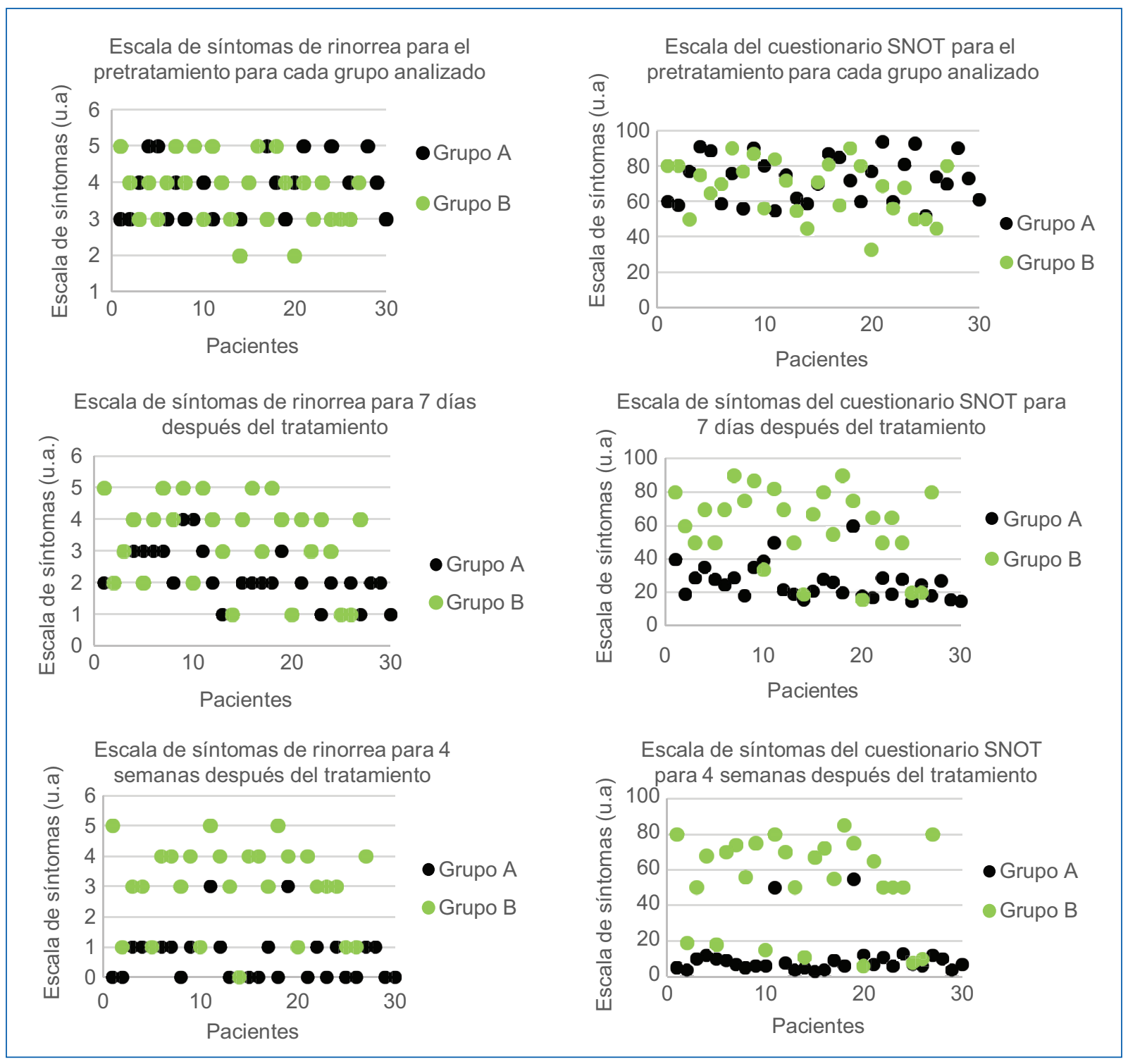

Figura 5. Comparativa entre los grupos A y B para el pretratamiento, 7 días y 4 semanas después del tratamiento, tanto para la escala de síntomas como para las respuestas del cuestionario SNOT (Sino-Nasal Outcome Test de 20 indicadores).

Después de cuatro semanas de tratamiento, el grupo A muestra que solo 2 pacientes, el $6.66 \%$ de ellos, indican una escala superior a 2 puntos, mientras que en el grupo B, el $74.07 \%$ de los pacientes refiere una escala superior a 2 puntos. Nuevamente, el mismo comportamiento se repite en las respuestas del cuestionario SNOT, donde en el grupo B, el $74.07 \%$ de los pacientes, manifiesta una escala de síntomas mayor a 40 puntos. Toda la información se resume en la tabla 2, donde se muestran los promedios y las desviaciones calculadas para cada grupo y periodo de tratamiento.
No hubo muertes ni eventos adversos graves con el uso del bromuro de ipratropio. Los eventos adversos más comunes relacionados al medicamento fueron sequedad e irritación nasal. La mayoría de los eventos fueron episodios simples de intensidad leve a moderada y resultó en la interrupción del medicamento en un paciente por irritación. En la figura 6 se muestran los resultados por sexo para cada grupo y tanto en hombre como en mujeres se observa el mismo comportamiento: una mejoría significativa con el uso del bromuro de ipratropio. Con un intervalo de confianza 

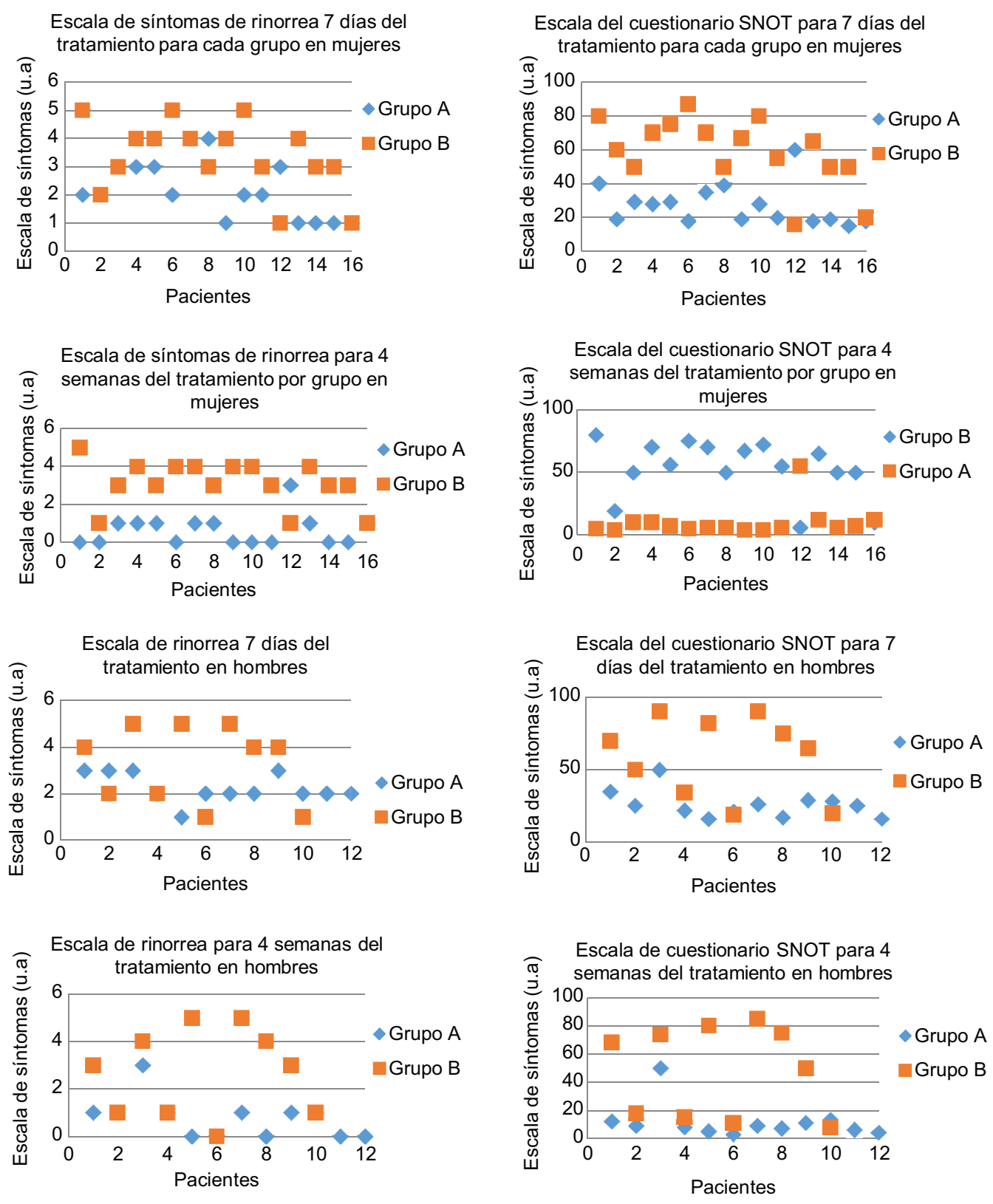

Figura 6. Escala de rinorrea y cuestionario SNOT (Sino-Nasal Outcome Test de 20 indicadores) para el periodo de tratamiento para cada grupo indicado por sexo.

Tabla 2. Promedios de la escala para el cuestionario rinorrea y SNOT (Sino-Nasal Outcome Test) manifestada por los pacientes de los grupos A y B

\begin{tabular}{|l|c|c|c|c|}
\hline \multirow{2}{*}{ Periodo } & \multicolumn{2}{|c|}{ Cuestionario rinorrea } & \multicolumn{2}{c|}{ Cuestionario SNOT } \\
\cline { 2 - 5 } & Grupo A & Grupo B & Grupo A & Grupo B \\
\hline Pretratamiento & $3.9 \pm 0.8030$ & $3.74 \pm 0.9026$ & $72.86 \pm 13.0773$ & $67.29 \pm 15.5633$ \\
\hline 7 días después del tratamiento & $2.16 \pm 0.8742$ & $3.37 \pm 1.3629$ & $26.2 \pm 10.5680$ & $3.00 \pm 1.4411$ \\
\hline 4 semanas después del tratamiento & $0.66 \pm 0.8022$ & $60 \pm 22.4293$ & $10.43 \pm 11.7903$ & $52.18 \pm 26.1916$ \\
\hline
\end{tabular}


Tabla 3. Cálculo de z

\begin{tabular}{|c|c|c|c|c|c|c|}
\hline \multicolumn{7}{|c|}{ Grupo A } \\
\hline & $\begin{array}{c}\text { Rinorrea } \\
\text { (pretratamiento) }\end{array}$ & $\begin{array}{l}\text { Rinorrea } \\
\text { (7 días) }\end{array}$ & $\begin{array}{c}\text { Rinorrea } \\
\text { (4 semanas) }\end{array}$ & $\begin{array}{c}\text { Cuestionario SNOT } \\
\text { (pretratamiento) }\end{array}$ & $\begin{array}{l}\text { Cuestionario } \\
\text { SNOT (7 días) }\end{array}$ & $\begin{array}{c}\text { Cuestionario } \\
\text { SNOT (4 semanas) }\end{array}$ \\
\hline$x$ & 4 & 2 & 1 & 70 & 30 & 10 \\
\hline$z$ & 0.1245 & -0.1906 & 0.4154 & -0.2192 & 0.3595 & -0.0367 \\
\hline$p$ & 0.5477 & 0.4246 & 0.6554 & 0.4207 & 0.6368 & 0.5119 \\
\hline \multicolumn{7}{|c|}{ Grupo B } \\
\hline & $\begin{array}{c}\text { Rinorrea } \\
\text { (pretratamiento) }\end{array}$ & $\begin{array}{l}\text { Rinorrea } \\
\text { (7 días) }\end{array}$ & $\begin{array}{l}\text { Rinorrea } \\
\text { (4 semanas) }\end{array}$ & $\begin{array}{c}\text { Cuestionario SNOT } \\
\text { (pretratamiento) }\end{array}$ & $\begin{array}{l}\text { Cuestionario } \\
\text { SNOT (7 días) }\end{array}$ & $\begin{array}{c}\text { Cuestionario } \\
\text { SNOT ( } 4 \text { semanas) }\end{array}$ \\
\hline$x$ & 4 & 2 & 1 & 70 & 30 & 10 \\
\hline$z$ & 0.2872 & -1.0054 & -1.3877 & 0.1737 & -1.3375 & -1.6106 \\
\hline $\mathrm{p}$ & 0.6102 & 0.1586 & 0.0837 & 0.5674 & 0.0917 & 0.0536 \\
\hline
\end{tabular}

SNOT: Sino-Nasal Outcome Test.

del $99 \%, p<0.001$, para el resto de los datos se muestra el cálculo de $\mathrm{Z}$ con la ecuación $\mathrm{Z}=(\mathrm{x}-\mu) / \sigma$, donde $\mathrm{x}$ es el valor buscado, $\mu$ es la media y $\sigma$ es la desviación (Tabla 3).

\section{Conclusiones}

El bromuro de ipratropio produjo reducciones estadística y clínicamente significativas en la gravedad y duración de la rinorrea en comparación con el placebo, además de mostrar una acción rápida en la disminución de síntomas, dado que después de siete días los pacientes manifestaron mejoría. Con el uso de la solución fisiológica no se mostró mejoría en los pacientes durante todo el estudio, lo cual queda demostrado tanto en los cuestionarios de rinorrea como en el cuestionario SNOT. El bromuro de ipratropio en dosis de $42 \mathrm{mcg}$ por cada fosa nasal tres veces al día fue bien tolerado por los pacientes durante el mes de estudio y aminoró los síntomas manifestados por los pacientes previo a su utilización. Con un intervalo de confianza del 99\%, $p<0.001$, siendo muy similar su eficacia, como lo reportado en la bibliografía, en comparación con el bromuro de ipratropio en su presentación de espray nasal. No se asoció con efectos secundarios adversos graves relacionados con el medicamento o clínicamente significativos para interrumpir el tratamiento, ni efectos sistémicos anticolinérgicos. No causó cambios perjudiciales en la mucosa nasal. Hubo una mejora en la calidad de vida de los pacientes, medida con el cuestionario SNOT antes, durante y al final del estudio. Por lo que se recomienda su uso como alternativa, al no contar con la presentación en nuestro país en espray nasal.

\section{Financiamiento}

Los autores no recibieron patrocinio para llevar a cabo este artículo.

\section{Conflicto de intereses}

Los autores declaran no tener conflicto de intereses alguno.

\section{Responsabilidades éticas}

Protección de personas y animales. Los autores declaran que los procedimientos seguidos se conformaron a las normas éticas del comité de experimentación humana responsable y de acuerdo con la Asociación Médica Mundial y la Declaración de Helsinki.

Confidencialidad de los datos. Los autores declaran que en este artículo no aparecen datos de pacientes.

Derecho a la privacidad y consentimiento informado. Los autores declaran que en este artículo no aparecen datos de pacientes.

\section{Bibliografía}

\footnotetext{
1. Georgitis JW, Banov C, Boggs PB, Dockhorn R, Grossman J, Tinkelman D, et al. Ipratropium bromide nasal spray in non-allergic rhinitis: efficacy, nasal cytological response and patient evaluation on quality of life. Clin Exp Allergy. 1994;24(11):1049-55.
} 
2. Kim KT, Kerwin E, Landwehr L, Bernstein JA, Bruner D, Harris D, et al.; Pediatric Atrovent Nasal Spray Study Group. Use of $0.06 \%$ ipratropium bromide nasal spray in children aged 2 to 5 years with rhinorrhea due to a common cold or allergies. Allergy Asthma Immunol. 2005;94:73-9.

3. Dockhorn R, Grossman J, Posner M, Zinny M, Tinkleman D. A dou ble-blind, placebo-controlled study of the safety and efficacy of ipratropium bromide nasal spray versus placebo in patients with the common cold. J Allergy Clin Immunol. 1992;90:1076-82.

4. Kaiser HB, Findlay SR, Georgitis JW, Grossman J, Ratner PH, Tinkelman DG, et al. The anticholinergic agent, ipratropium bromide, is useful in the treatment of rhinorrhea associated with perennial allergic rhinitis. Allergy Asthma Proc. 1998;19:23-9.

5. Sjögren I, Jonsson L, Köling A, Jansson C, Osterman K, Håkansson B. The effect of ipratropium bromide on nasal hypersecretion induced by methacholine in patients with vasomotor rhinitis. Acta Otolaryngol. 1988;106:453-9.

6. Borum P. Intranasal ipratropium: inhibition of methacholine-induced hypersecretion. Rhinology. 1978;16:225-33.

7. Malmberg H, Grahne B, Holopainen E, Binder E. Ipratropium (Atrovent) in the treatment of vasomotor rhinitis of elderly patients. Clin Otolaryngo Allied Sci. 1983;8(4):273-6.
8. Kaiser HB, Findlay SR, Georgitis JW, Grossman J, Ratner PH, Tinkelman DG, et al. Long-term treatment of perennial allergic rhinitis with ipratropium bromide nasal spray $0.06 \%$. J Allergy Clin Immunol. 1995:95(5 Pt 2):1128-32.

9. Mygind N, Weeke B. Allergic and nonallergic rhinifis. En: Middleton E, Reed, Elis E, editores. Allergy, principles and practice. St. Louis: CV Mosby; 1983. pp. 1107-1117.

10. Dockhorn R, Aaronson D, Bronsky E, Chervinsky P, Cohen R, Ehtessabian $\mathrm{R}$, et al. Ipratropium bromide nasal spray $0.03 \%$ and beclomethasone nasal spray alone and in combination for the treatment of rhinorrhea in perennial rhinitis. Ann Allergy Asthma Immunol. 1999;82(4): 349-59.

11. Naclerio RM, Baroody FM. In vivo human models for the study of anticholinergic drugs. J Allergy Clin Immunol. 1995;95:1069-79.

12. Magardino TM, Suskind-Liu D, Kroger H, Mirza N. Ipratropium bromide nasal spray for treatment of rhinorrhea in the laryngectomized patient: A pilot study. Am J Rhinol. 2001;15(3):203-6.

13. Graf $P$, Eccles R, Chen S. Efficacy and safety of intranasal xylometazoline and ipratropium in patients with common cold. Expert Opin Pharmacother. 2009;10(5):889-908.

14. Brożek JL, Bousquet J, Agache I, Agarwal A, Bachert C, Bosnic-Anticevich $S$, et al. Allergic Rhinitis and its Impact on Asthma (ARIA) guidelines-2016 revision. J Allergy Clin Immunol. 2017;140(4):950-8. 